\title{
Longitudinal Cortical Registration for Developing Neonates
}

\author{
Hui Xue ${ }^{1,2}$, Latha Srinivasan ${ }^{1,2,3}$, Shuzhou Jiang ${ }^{1}$, Mary Rutherford ${ }^{1}$, \\ A. David Edwards ${ }^{1,3}$, Daniel Rueckert ${ }^{2}$, and Joseph V. Hajnal ${ }^{1}$ \\ ${ }^{1}$ Imaging Sciences Department, Imperial College, London, Du cane Road, W12 0NN, UK \\ ${ }^{2}$ Department of Computing, Imperial College, London, 180 Queen's Gate, SW7 2BZ, UK \\ ${ }^{3}$ Department of Paediatrics, Imperial College, London, Du cane Road, W12 0NN, UK \\ \{hui.xue, 1.srinivasan, shuzhou.jiang, mary.rutherford, \\ david.edwards, d.rueckert, jo.hajnal\}@imperial.ac.uk
}

\begin{abstract}
Understanding the rapid evolution of cerebral cortical surfaces in developing neonates is essential in order to understand normal human brain development and to study anatomical abnormalities in preterm infants. Several methods to model and align cortical surfaces for cross-sectional studies have been developed. However, the registration of cortical surfaces extracted from neonates across different gestational ages for longitudinal studies remains difficult because of significant cerebral growth. In this paper, we present an automatic cortex registration algorithm, based on surface relaxation followed by non-rigid surface registration. This technique aims to establish the longitudinal spatial correspondence of cerebral cortices for the developing brain in neonates. The algorithm has been tested on 5 neonates. Each infant has been scanned at three different time points. Quantitative results are obtained by propagating sulci across multiple gestational ages and computing the overlap ratios with manually established ground-truth.
\end{abstract}

\section{Introduction}

Clinical studies have shown delayed cortical folding and white matter (WM) related macro- and micro-structural changes in preterm infants at term equivalent age [1]. By analyzing changes in the neonatal cortex during early phases of brain development, it may be possible to detect precursors of cerebral abnormalities prior to term equivalent age, which would allow treatment options to be tested during the neonatal period. Also, the rapid anatomical and functional evolution of neonatal cortex itself presents a major mystery for evolutionary biologists and neuroscientists. Cortical development during the third trimester of pregnancy is extensive with noticeable increase in the cortical folding. In addition there is significant cortical variability across infants. Thus, the precise localization and tracking of principal anatomical features, i.e. central sulcus and sylvian fissure, is difficult.

Several researchers have presented algorithms to unfold and align the cerebral cortex in cross-sectional studies in adulthood [2, 3, 4, 5]. Methods based on cortex unfolding aim to inflate the highly folded surfaces and map the whole or a piece of cortex to some standard representations like a flat surface or a sphere. The inflation 
process is normally regularized by ensuring that several constraints, like rigidity between neighboring points, or the local area and angle are minimally distorted during the unfolding procedure [3]. The alignment of corresponding anatomical features is partly achieved by identifying these features manually and then normalizing the sphere into a standard coordinate space $[4,5]$. This requirement of maintaining strict point correspondences was recently relaxed by Tosun et al. [6]. They applied a rigid surface registration to remove global misalignment between two cortical surfaces before applying a conformal mapping to transform them to a spherical representation. They also showed that using a normalized spherical coordinate system the four main sulci can be aligned across individuals. Although some measurements, such as surface area and distance can be computed with the spherical coordinate normalized by a rigid body transformation, this representation tends to smooth out fine-grain details of complex cortical anatomy. However, in the developing brain, specific sulci can experience significant morphometric development during the third trimester of pregnancy. Also, a global rigid body transformation clearly is not able to capture local non-rigid deformation. Instead a non-rigid registration is required to follow the growth of specific sulci across gestational ages (GAs).

The aim of this study is to develop a methodology which is able to track and quantify cortical development in neonates longitudinally and to evaluate its ability to identify cerebral abnormalities related to preterm birth. To enable the tracking of cortical development we have developed a cortical registration algorithm based on two stages: In the first stage the more mature cortex (e.g. the later time point) is progressively smoothed. This smoothing process is repeated until the more mature cortex is maximally similar to the less mature cortex. In the second stage any residual misalignment of the cortex is corrected by performing a non-rigid surface registration using free-form deformations (FFDs). A quantitative evaluation of the cortical registration is performed by propagating sulci across multiple gestational ages and computing the overlap ratios with manually established ground-truth.

\section{Methods}

\subsection{Cortical Segmentation from Neonatal MR Images}

The automatic segmentation of cortical grey matter in neonatal MRI is more challenging than the segmentation of adult brains. A particular confounding factor is the inverted white matter (WM) and gray matter (GM) contrast compared to the adult pattern. This leads to mislabeled voxels at the interface between the cerebrospinal fluid (CSF) and GM. Because CSF has the highest intensity in neonatal T2w images and the image resolution of neonatal MRI is usually no more than $0.9 \mathrm{~mm}^{3}$, many voxels between CSF and GM will have similar intensities to WM which is brighter than GM and darker than CSF (Fig. 1a). These voxels can be incorrectly classified as WM by conventional intensity-based segmentation approaches (Fig. 1b). We have developed an automated cortical segmentation algorithm addressing these difficulties [7]. Specifically, a modified expectation-maximization (EM) scheme is used in combination with a Markov Random Field (MRF) model to ensure spatial homogeneity in the tissue classification. The detection and removal of mislabeled partial volume voxels (MLPV) is based on a knowledge-based rule. The MLPV are 


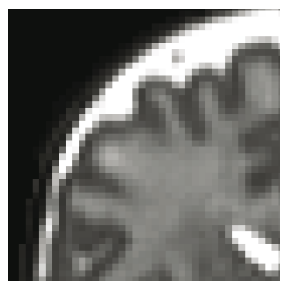

(a)

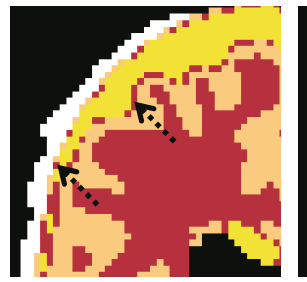

(b)

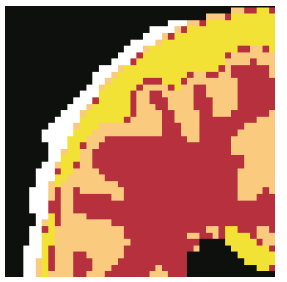

(c)

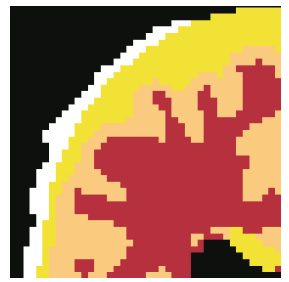

(d)

Fig. 1. An illustration of neonatal cortex segmentation. (a) An enlarged neonatal T2w image; Note the WM is brighter than GM. (b) Partial volume voxels (highlighted by arrows) on the CSF-GM and CSF-non-brain boundaries are incorrectly classified by the original EM method; (c) The segmentation results after the 4-th iteration; (d) The final results after 14 iterations.

identified after every EM iteration and the MRF priors are adjusted to favor the correct classification classes. Once the modified EM algorithm converges most of the MLPV are eliminated (Fig. 1c).

\subsection{Cortical Surface Reconstruction}

Starting from a probabilistic tissue classification generated by the automated segmentation algorithm [7], we reconstructed the inner, central and outer cortical surfaces for neonates using the reconstruction algorithm proposed in [8]. Specifically, the cortical surfaces are implicitly represented by the zero level-set and surface evolution is driven by solving the standard level-set partial differential equation.

\subsection{Cortical Surface Registration}

Each reconstructed cortex is represented as a polygon mesh consisting of between 35,000 and 90,000 triangles. We do not introduce an explicit unfolding step to map the cortical surfaces to spherical representation. On the contrary, the registration method is designed to establish point correspondences on the original surfaces.

As the first step we remove any global affine misalignment between the two cortical surfaces. We have found that direct alignment of the cortical surfaces by the simple iterative closest point (ICP) method often converges to local minima, whereas voxel-based image registration [9] is much more robust and can deal with significant global affine differences between the neonatal brains. Thus, the corresponding anatomical T2 weighted images are firstly registered and the optimal affine transformation obtained is then applied to the polygon meshes.

Neonatal cerebral cortex normally undergoes rapid changes and the complexity of cortical folding increases noticeably during the gestational ages from between $\sim 27$ to 45 weeks. This rapid development process makes it difficult to establish correspondences directly via non-rigid surface registration. We found that smoothing the more mature cortex by reducing the complexity of the folding patterns generates cortical surfaces which are much more similar to the cortex obtain from early scans during cortical development. Thus, our assumption is that the performance of a cortical surface registration algorithm for aligning longitudinal data can be improved by exploiting this fact. We therefore perform an adaptive surface relaxation step 
before performing the non-rigid surface registration. It is also inspired by the observation in [6], where it was reported that partially smoothed surfaces can lead to more consistent spherical maps.

Adaptive surface relaxation. Surface relaxation has been originally designed to smooth reconstructed polygon surfaces and to reduce artifacts which often appear as abrupt or stair-step meshes. The relaxation process is helpful for improving visualization $[10,11]$. To facilitate any non-rigid surface registration, we here employ surface relaxation prior to the surface registration to inflate the more mature cortex.

One iteration step of this relaxation process is defined as follows $[11,12,6]$ :

$$
\boldsymbol{v}_{t+1}^{i}=(1-\lambda) \cdot \boldsymbol{v}_{t}^{i}+\lambda \cdot \overline{\boldsymbol{v}}_{t}^{i}
$$

where $\boldsymbol{v}_{t}^{i}$ is the position of vertex $i$ at the iteration $t . \lambda \in[0,1]$ is a pre-defined smoothing factor. $\overline{\boldsymbol{v}}_{t}^{i}$ is the average vertex position of all polygons sharing vertex $i$ :

$$
\overline{\boldsymbol{v}}_{t}^{i}=\frac{1}{\sum_{j \in N_{i}} A_{j}} \sum_{j \in N_{i}} A_{j} \cdot \boldsymbol{C}_{j}
$$

where $N_{j}$ is the set of polygons using the vertex $i \cdot A_{j}$ and $C_{j}$ are the surface area and centre of polygon $j$.

It is necessary to define a stopping criterion for surface relaxation, so that the cortical folding complexity of the more mature surface is comparable to the folding complexity seen at the earlier gestational age. We have tested various cortical folding measures. In this paper we have decided to use a criterion that is based on the computation of the intrinsic curvature index $(I C I)$ and mean curvature $L^{2}$ norm $(M L N)$. Both measures are dimensionless and measure different aspects of cortical folding complexity. The former is originally defined in [13], measuring the local intrinsic convexity of surface. The MLN is the $L^{2}$ norm of the mean curvature of cortical surfaces which takes a minimum value for a sphere and is called bending energy [14].

$$
I C I=\int_{S}\|K\|_{+} d A \quad M L N=\sqrt{\int_{S} H^{2} d A}
$$

where $S$ is the whole cortical surface. $K$ and $H$ are Gaussian and mean curvature. $\|K\|_{+}$equals $K$ if $K>0$ and otherwise it is zero. Both measures are integrated over the whole cortical surface. The relaxation will stop when both ICI and MLN of inflated surface fall below the corresponding values for the less mature cortex. An illustration of this surface relaxation is given in Figure 2.

Non-rigid surface registration based on free-form deformations (FFDs). The output of the adaptive surface relaxation is finally registered to the less mature cortical surface using a non-rigid surface registration algorithm. We use an algorithm based on free-form deformations (FFDs) which is a powerful tool for modeling 3-D deformable objects [15]. The basic idea of FFDs is to deform an object by manipulating an underlying mesh of control points. The resulting deformation controls the shape of the 3-D object and remains as a $C^{2}$ continuous transformation, which smoothly deforms the cortical surfaces. 


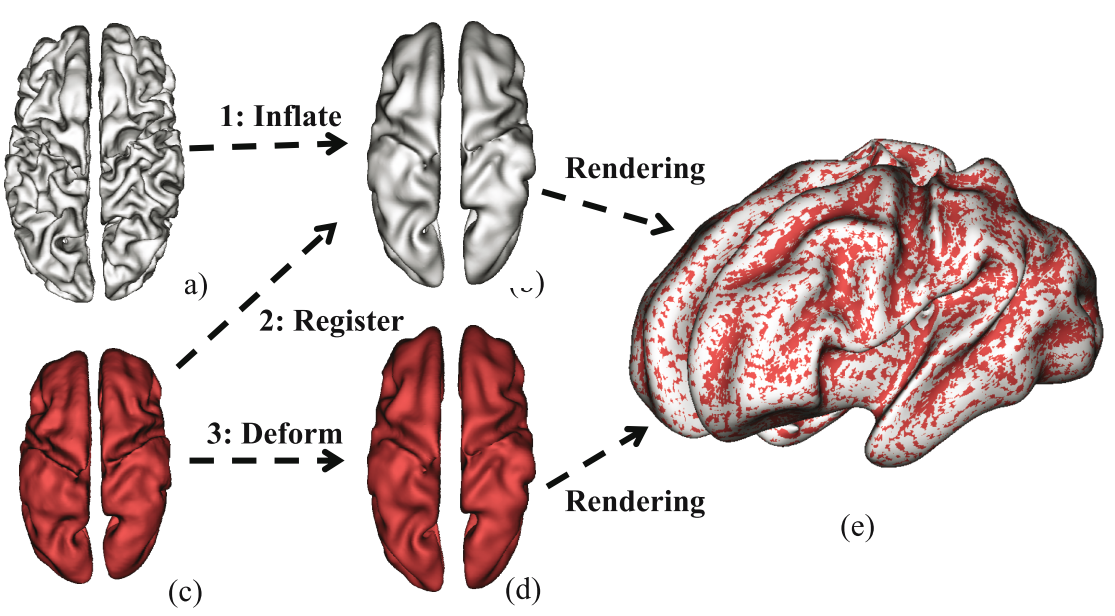

Fig. 2. An illustration of cortex surface relaxation and non-rigid registration. A neonate was scanned for three times. The inner cortical surface of the first scan (GA: 29.86 weeks) is shown in (c). The cerebral cortex has undergone noticeable development by the scan at term equivalent age (GA: 39.86 weeks), as shown in (a). The inflated surface after adaptive relaxation is shown in (b), where the cortical folding complexity is substantially decreased. Non-rigid surface registration is performed to align the less mature cortex (c) and inflated surface (b). The deformed surface of (c) is shown in (d). (e) renders (b) and (d) together. The zigzag pattern shows these two surfaces are spatially very close.

To define a FFD for a cortical surface $S$, we define the spatial domain occupied by this surface as follows: $\Omega_{s}=\{(x, y, z) \mid 0 \leq x \leq X, 0 \leq y \leq Y, 0 \leq z \leq Z\}$ and $\phi_{s}$ denotes a $n_{x} \times n_{y} \times n_{z}$ grid of control points $\varphi_{i, j, k}$. The spacing between adjacent control points is uniform in all coordinate directions. The deformation of a vertex $\boldsymbol{v}_{i}=(x, y, z)$ is represented as the 3D tensor of the 1-D cubic B-splines [15]:

$$
\boldsymbol{T}_{\text {local }}\left(\boldsymbol{v}_{i}\right)=\sum_{l=0}^{3} \sum_{m=0}^{3} \sum_{n=0}^{3} B_{l}(u) B_{m}(v) B_{n}(w) \varphi_{i+l, j+m, k+n}
$$

where $i=\left\lfloor x / n_{x}\right\rfloor-1, j=\left\lfloor y / n_{y}\right\rfloor-1, k=\left\lfloor z / n_{z}\right\rfloor-1, u=x / n_{x}-\left\lfloor x / n_{x}\right\rfloor, v=y / n_{y}-\left\lfloor y / n_{y}\right\rfloor$ and $w=z / n_{z}-\left\lfloor z / n_{z}\right\rfloor . B_{l}$ represents the $l$-th basis function of the B-spline. The basis functions of cubic B-splines have limited support. Therefore changing a control point in the grid affects only a $4 \times 4 \times 4$ region around that control point. Surface registration is achieved by specifically moving the control points to minimize a surface similarity. The similarity which we try to optimize is the average symmetric spatial distance $f$ :

$$
f\left(\boldsymbol{S}, \boldsymbol{W}, \boldsymbol{T}_{\text {local }}\right)=\frac{1}{N_{S}} \sum_{i=1}^{N_{S}}\left\|\boldsymbol{v}_{i}-\ell\left(\boldsymbol{v}_{i}, T_{\text {local }}(\boldsymbol{W})\right)\right\|_{2}+\frac{1}{N_{W}} \sum_{j=1}^{N_{W}}\left\|\boldsymbol{w}_{j}-\ell\left(\boldsymbol{w}_{j}, \boldsymbol{S}\right)\right\|_{2}
$$

where $\boldsymbol{S}$ and $\boldsymbol{W}$ are two cortical surfaces which are being registered. $N_{S}$ is the number of vertexes in surface $S . N_{W}$ is the number of vertexes in surface $\boldsymbol{W}$. For 
every vertex $\boldsymbol{v}_{i} \in \boldsymbol{S}, \ell\left(\boldsymbol{v}_{i}, \boldsymbol{T}_{\text {local }}(\boldsymbol{W})\right)$ defines the closest vertex of $\boldsymbol{v}_{i}$ on the transformed surface $\boldsymbol{T}_{\text {local }}(\boldsymbol{W})$. Similarly, for every vertex $\boldsymbol{w}_{j} \in T_{\text {local }}(\boldsymbol{W}), \ell\left(\boldsymbol{w}_{j}, \boldsymbol{S}\right)$ defines the closest vertex of $\boldsymbol{w}_{j}$ on the surface $S$. The purpose of adding the second item is to force the registration of deep sulci. To ensure that the spatial transformation defined by the FFD is smooth, a standard second order regularization penalty should be minimized [16]. This penalty is added to the surface similarity to produce the final cost function.

\section{Results and Evaluation}

We applied our method to 15 images acquired from 5 neonates. These infants are selected from a longitudinal MR study of cerebral development of premature neonates. Every subject has had three longitudinal scans. The initial scans were performed between 27 weeks and 33 weeks gestational age. The second scans were performed at a mean GA of 35 weeks and the final images were acquired at the term equivalent age (mean of 41 weeks).

MR images were acquired on a 3T Philips Intera system (Best, Holland). The MR sequence parameters were as follows: T2-weighted fast spin echo pseudo volumes: $\mathrm{TR}=1712 / \mathrm{TE}=160 \mathrm{~ms}, \mathrm{FOV}=220 \mathrm{~mm}$, matrix $224 \times 224$, flip angle $90^{\circ}$, voxel size of $0.86 \times 0.86 \times 2 \mathrm{~mm}$ with the $50 \%$ slice overlap. After acquisition, the T2 images were segmented using the algorithm described in section 2.1 and the inner cortical surfaces were reconstructed and used for registration.

Fig. 2 shows an example of the surface relaxation and cortical registration results between longitudinal scans. The more mature cortex is relaxed until its folding complexity is comparable to the less mature cortex. When working with the registration between two later scans (scans of 35 weeks and 41 weeks) where folding patterns are becoming more complex, we found that inflating $\sim 35$ weeks surfaces can improve the registration. Those cortexes were therefore smoothed until their ICI and $M L N$ decreased by $15 \%$. The $\sim 41$ weeks surfaces were then adaptively inflated to match folding measurements from previous time points.

We have performed cortical registration to register the cortex between subsequent time points for all subjects. Also, we have registered the cortical surface at the first time point directly to the cortical surface at the last time point (term-equivalent age) which is more challenging due to the significant cortical development during this time interval. To quantify the ability of the proposed registration method to localize and track the main anatomical features of the cortex, an experienced neonatologist was asked to manually label the central sulci (CS) on all 15 cortical surfaces. The manual labeling of one cortex can then be mapped to its target surface and generate an automatic segmentation via the non-rigid deformation. Fig. 3 gives an illustration of this automatic sulcus labeling. Note that a significant amount of non-rigid deformation is required to map the central sulcus extracted from less mature cortices to later scans. This deformation itself can be used to describe the local evolution of neonatal cortices, which may not be explicitly represented using spherical mapping.

The overlap between automated results and manually established ground-truth were computed as a quantitative measurement of registration performance. Both true positive (TP) and false positive (FP) errors are estimated. TP is computed as the percentage area of manual labeling that is accurately labeled by the automatic 
mapping. FP is defined as the percentage area of automatic sulcus that is not labeled manually. Table 1 summarizes the results. In all cases, the cortical registration with surface inflation shows the best performance. It is also clear that performing just global affine transformation is not sufficient for automated sulcus mapping. Direct non-rigid surface registration shows higher error rates possibly because the more folds a cortical surface presents, the more local optima the surface similarity can have.

Table 1. Mean overlap ratios of automatic central sulcus labelling

\begin{tabular}{c|c|c|c|c|c|c|c|c|c|c|c|c}
\hline & \multicolumn{4}{|c|}{ 1st to 2nd } & \multicolumn{4}{c|}{ 2nd to 3rd } & \multicolumn{4}{c}{ 1st to 3rd } \\
\cline { 2 - 26 } & Affine & IM & NR & NR+I & Affine & IM & NR & NR+I & Affine & IM & NR & NR+I \\
\hline TP & 0.16 & 0.81 & 0.73 & 0.97 & 0.20 & 0.77 & 0.71 & 0.91 & 0.07 & 0.31 & 0.41 & 0.72 \\
\hline FP & 0.76 & 0.15 & 0.14 & 0.12 & 0.71 & 0.25 & 0.08 & 0.11 & 0.87 & 0.71 & 0.41 & 0.27 \\
\hline
\end{tabular}

- 1st to 2nd: mapping central sulcus from the first scan to the second scan; 2nd to 3rd and 1st to 3nd are similarly defined;

- Affine: global affine transformation; IM: intensity based non-rigid registration; NR: only non-rigid surface registration; NR+I: non-rigid surface registration with adaptive surface inflation.

\section{Discussion and Conclusions}

One aim of cortical unfolding techniques developed for adult brains is to normalize an intermediate representation, i.e. sphere or flat, so that primary anatomical features can appear at the same coordinates in the mapped space. This allows comparison of different patient groups. However the neonatal cortical evolution presents a somewhat different challenge because of evolving complexity with gestational age and it provides a unique opportunity to understand normal human brain development both at an individual level and evolutionary level. Therefore we developed a non-rigid surface registration technique and tested its applicability for use on MR images of developing neonates. Here we have shown that using this technique we can capture local deformations, and track and localize the central sulcus across different gestational ages.

The preliminary evaluation in this paper shows that the non-rigid registration achieves better performance if the cortical surfaces are partially inflated. We hypothesize that partial inflation reduces the likelihood of the algorithm stopping in local optima of the surface similarity measure. This aids registration performance. However, the inflation can smooth out the secondary sulci and other smaller features, which can limit the non-rigid surface deformation to only capture changes in the main sulci. This side-effect might be reduced by designing a knowledge based surface similarity measure based on sulcus shape. Improving the surface initialization method may also reduce the degree of inflation needed for effective non-rigid registration. In the future we would like to apply this type of registration technique to both fetal and neonatal brains at differe gestational ages to aid the development of atlases of normal cortical growth patterns so that temporal events in altered cortical development of preterm infants can be identified. 


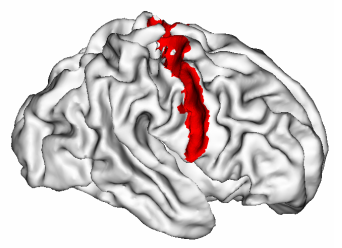

(a)

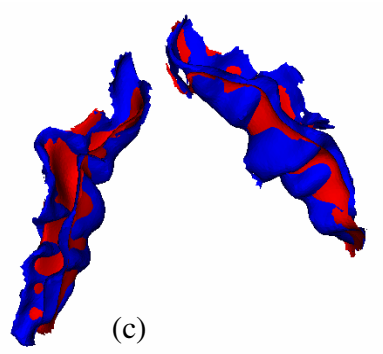

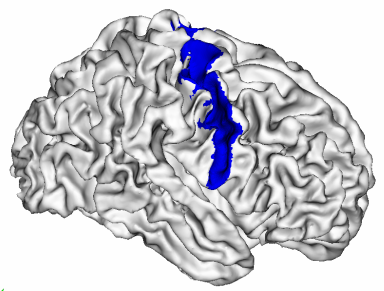

(b)

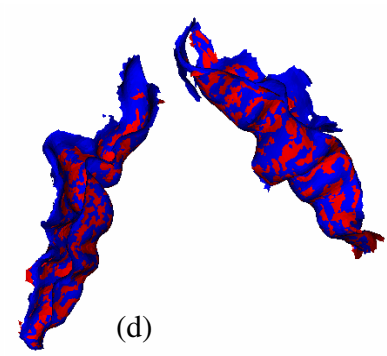

Fig. 3. Automatic sulcus labelling via non-rigid cortical registration. A neonate was scanned three times. (a) The inner cortex of the second scan (GA: 33.86 weeks); (b) the cortex at term equivalent age (GA: 39.86 weeks). Their central sulci are manually labelled. (c) Global affine transformation can not deform the central sulcus across time points, while the non-rigid surface registration captures the local evolution.

\section{References}

1. Kapellou, O., Counsell, S.J., Kennea, N.: Abnormal cortical development after premature birth shown by altered allometric scaling of brain growth. PLOS Medicine 3(8), 1382 1390 (2006)

2. Carman, G.J., Drury, H.A., van Essen, D.C.: Computational methods for reconstructing and unfolding the cerebral cortex. Cerebral Cortex 5(6), 506-517 (1995)

3. Fischl, B., Sereno, M.I., Dale, A.M.: Cortical surface-based analysis II: Inflation, flattening, and a surface-based coordinate system. NeuroImage 9(2), 195-207 (1999)

4. Thompson, P.M., Woods, R.P., Mega, M.S., Toga, A.W.: Mathematical/computational challenges in creating deformable and probabilistic atlases of the human brain. Human Brain Mapping 9, 81-92 (2000)

5. Gu, X., Wang, Y., Chan, T.F., Thompson, P.M., Yau, S.T.: Genus zero surface conformal mapping and its application to brain surface mapping. In: Taylor, C.J., Noble, J.A. (eds.) IPMI 2003. LNCS, vol. 2732, pp. 172-184. Springer, Heidelberg (2003)

6. Tosun, D., Rettmann, M.E., Prince, J.L.: Mapping techniques for aligning sulci across multiple brains. Medical Image Analysis 8(3), 295-309 (2004)

7. Xue, H., Srinivasan, L., Jiang, S.Z., Rutherford, M., Edwards, A.D., Rueckert, D., Hajnal, J.V.: Automatic Cortical Segmentation in the Developing Brain. In: Information Processing in Medical Imaging, pp. 257-269 (2007)

8. Han, X., Pham, D.L., Tosun, D., Rettmann, M.E., Xu, C., Prince, J.L., CRUISE,: Cortical reconstruction using implicit surface evolution. NeuroImage 23, 997-1012 (2004)

9. Rueckert, D.: Image Registration Toolkit., wwwhomes.doc.ic.ac.uk/ dr/software

10. MacDonald, D., Kabani, N., Avis, D., Evans, A.: Automated 3D extraction of inner and outer surfaces of cerebral cortex from MRI. NeuroImage 12(3), 340-356 (2000)

11. Drury, H.A., Essen, D.C.V., Anderson, C.H., Lee, W.C., Coogan, T.A., Lewis, J.W.: Computerized mapping of the cerebral cortex: a multiresolution flattening method and a surface-based coordinate system. J. Cogn. Neuro. 8(1), 1-28 (1996) 
12. Timsari, B., Leahy, R.: Optimization method for creating semiisometric flat maps of the cerebral cortex. In: Proceedings of the SPIE Conference on Medical Imaging: Image Processing, pp. 698-708 (2000)

13. Van Essen, D.C., Drury, H.A.: Structural and Functional Analyses of Human Cerebral Cortex Using a Surface-Based Atlas. The Journal of Neuroscience 17(18), 7079-7102 (1997)

14. Batchelor, P.G., Castellano Smith, A.D., Hill, D.L.G., Hawkes, D.J., Cox, T.C.S., Dean, A.F.: Measures of folding applied to the development of the human fetal brain. IEEE Transactions on Medical Imaging 21(8), 953-965 (2002)

15. Lee, S., Wolberg, G., Chwa, K.-Y., Shin, S.Y.: Image metamorphosis with scattered feature constraints. IEEE Trans. Visualization Comput. Graph. 2, 337-354 (1996)

16. Wahba, G.: Spline models for observational data. Soc. Industr. Applied Math. (1990) 